\title{
Dispositivos estructurales de la acción colaborativa en contextos de integración regional
}

\section{Miguel Ángel Recondo ${ }^{1}$}

INAP-Universidad del Salvador

Tipo de trabajo: Artículo

Material original autorizado para su primera publicación en el Journal de Ciencias Sociales, Revista Académica de la Facultad de Ciencias Sociales de la Universidad de Palermo, dado que los derechos sobre el material publicado, para ediciones posteriores los reserva el autor.

Recibido: 19-9-2014

Aceptado: $13-3-2015$

\section{RESUMEN}

Los hábitos colaborativos se sostienen en mecanismos de conducta colectiva observables en la construcción de ciudadanía; desde el individuo activo, desplegándose en los espacios organizacionales intermedios y llegando al nivel de decisiones macro, nacionales y regionales. Postulamos que es imprescindible poder registrar y mostrar casos germinales de conductas colaborativas en los diversos espacios de construcción institucional, si deseamos aspirar a un reto tan desafiante como es la integración entre países. La metodología utilizada es el análisis de casos, aplicada al campo de la innovación tecnológica e institucional, tomando dos procesos relevantes: el distrito industrial del municipio de Santa Rita, Estado de Minas Gerais, Brasil, que incluye al Instituto de Telecomunicaciones- INATEL y su Polo Científico y Tecnológico, y el sistema universitario- tecnológico del Estado de Georgia, en EUA y su iniciativa Yamacraw, y su despliegue en las últimos 20 años. Como principales resultados se describen las organizaciones flexibles, con capacidad de reproducirse en un sistema suprarregional - fractales- y los drivers necesarios para

\footnotetext{
${ }^{1}$ Doctor en Relaciones Internacionales. Coordinación de redes y articulación territorial, institucional y sectorial. INTI - Área Trabajo y Educación a Distancia.

miguelrecondo@gmail.com
} 
una construcción colaborativa en el mismo espacio geográfico, aplicados a los sectores productivo - científico-tecnológico- educativo en espacios nacionales, y su validación en los casos estudiados. En el espacio de discusión, se propone abrir los campos del análisis de la cultura y la política así como el reto de construir conceptualizaciones y nuevos significados para las experiencias diversas de nuestro continente. En las conclusiones sostenemos que las organizaciones complejas estructuradas en redes y dispositivos colaborativos constituyen instrumentos imprescindibles para los procesos de integración regional.

\section{Palabras claves}

Enfoque sistémico. Organización Fractal. Contextos. Redes de aprendizaje.

Dispositivos de colaboración. Integración regional.

\section{ABSTRACT}

This article presents the study of two cases of technological and institutional innovation: the industrial district of Santa Rita, Estado de Minas Gerais, Brasil, that considers the Instituto de Telecomunicaciones (INATEL) with its scientific and technology pole and the technology and university system of Georgia State (USA) with the Yamacraw initiative. The results shows that complex organizations organized in networks and collaboration are necessary for the development of processes of regional integration.

\section{Palabras claves:}

Regional Integration, Collaborative Networks, Organizations, Technology.

\section{Introducción}

\section{a. Perspectiva de abordaje.}

Los procesos de integración regional (IR) emergen en el siglo XX como unos de los paradigmas necesarios para el despliegue de las naciones en un mundo donde la escala de los actores políticos y socioeconómicos es determinante.

En tanto la inquietud que sostiene este trabajo es la necesidad que tiene Latinoamérica de completar un proceso de integración regional, su objetivo es desplegar una descripción de la funcionalidad de los dispositivos colaborativos en la construcción de procesos de integración nacional y continental (IC), en el espacio vincular de la tecnología y la educación superior interactuando con el mundo 
productivo. En tanto, su dinámica es vista y analizada desde el paradigma sistémico de la complejidad, donde las acciones registradas en algunos de los subsistemas -micro, meso o macro- impactan en los dos restantes.

Hacia esta finalidad, el componente que surge como herramienta central es la cultura del trabajo colaborativo en todos los espacios comunitarios. Surgen entonces preguntas del tipo: ¿en qué grado existe esa cultura en los países involucrados en un proceso de IR? Y en segundo lugar, ¿existen experiencias de suficiente consistencia que puedan tomarse como referencias modelo para activar procesos integradores en escala regional?

Las perspectivas más transitadas son las que problematizan las políticas macro de intervención de los estados nacionales en la construcción del proceso de integración, especialmente dirigidas al sistema económico y productivo, como el caso del Mercosur. Naturalmente, este es un recorrido top-down, atendiendo a consensuar normativas y políticas desde la cima del Estado- su gobierno -, hacia abajo previendo ventajas y desventajas y gobernabilidad del sistema como un todo. (Best, 1993)

Mucho menos aplicado es el enfoque de construcción "desde abajo", que prioriza al ciudadano o al agente institucional que desea alcanzar metas en beneficio de la comunidad. Es este hombre/ institución - y su innegable potencial - a quien tenemos presente en su función de observar y actuar colaborativamente desde su acción posible de cada día.

En consecuencia, para impulsar y consolidar procesos de IR se debe verificar la presencia de las capacidades necesarias en los distintos planos de construcción ciudadana; desde el nivel micro de cada individuo, desplegándose en los espacios organizacionales intermedios y llegando al nivel de decisiones macro, nacionales y regionales. Postulamos que es imprescindible poder registrar y mostrar casos germinales de conductas colaborativas en los diversos espacios de construcción institucional, si deseamos aspirar a un reto tan desafiante como es la integración entre países.

Abordar esta temática implica, a la vez, explicitar la perspectiva cognoscitiva de análisis- el espacio epistemológico- desde el cual trabajaremos y el tipo de gestión del conocimiento que utilizaremos, es decir, el conjunto de metodologías, constructos y lecturas de los datos reunidos. En esta línea de trabajo profundizaremos en la estructura y alcances del constructivismo y en el pensamiento sistémico como herramienta principal de nuestro análisis. Posteriormente explicitaremos a través de la metodología de casos, procesos que, en diversa extensión, dan cuenta de la plausibilidad y 
validación de nuestro enfoque.

Para ello, establecemos que para actuar de modo colaborativo - y todas sus variantes de cooperación y alianzas- es necesario identificar y generar un conjunto de organizaciones complejas, a saber, organizaciones fractales -referentes de un sistema - un tipo de ellas: las redes de vinculación y aprendizaje y drivers o interfaces instrumentales, que deben desplegarse en los niveles micro- el de los actores/agentes- y en el nivel meso; de las unidades institucionales en las cuales activan los agentes.

\section{b. El estado del arte}

Los enfoques predominantes hasta ahora relacionados con la activación de procesos de IR han puesto el acento en la dimensión macro de las políticas nacionales y, como primer paso, la importancia de iniciar procesos de integración económica creciente, siguiendo los ejemplos más conocidos de integración.

En lo que hace a la literatura actual y a las diversas teorías que intentan explicar y prescribir futuros pasos de este proceso se encuentran variantes que acentúan el espacio político-organizacional y la dimensión de la gobernabilidad (Christian von Haldenwang, 2005), el valor del pensamiento sistémico y el neofuncionalismo con temas tales como la complementariedad de las élites y el federalismo (Oyarzún Serrano, Lorena, 2008). Oyarzún también da relevancia a Karl Deutch y Antje Wiener. El enfoque de Deutch es precursor de los estudios de integración regional y referente principal de las Teoría de las comunicaciones. Para los transaccionalistas "la unión política se explica en función del flujo de comunicaciones (transacciones) que se establece dentro de un territorio y de éste con el mundo exterior". También es mencionado el trabajo de Antje Wiener y su afinidad con el constructivismo en el sentido que aplicamos en este trabajo. En el caso del señalamiento del enfoque sistémico, se trabaja sobre su método, pero no se profundiza en la dinámica de las realidades a las que apuntamos en este escrito. Sintetizando, vemos que el enfoque que aquí sostenemos está iniciado por algunos autores que, a su vez, invitan a enriquecer sus aportes.

\section{Perspectiva teórica.}

En nuestro caso hemos orientado el análisis hacia la dinamización operativa y sistémica de la IR, y la importancia de las diferentes partes de un sistema, la capacidad de sinergia entre ellas y la posibilidad de poner en valor experiencias limitadas en un espacio - micro, meso o macro - que puede tener su propia aplicabilidad y expansión en los dos restantes. Este enfoque posee la virtud 
de poder pensar acciones y estrategias no sólo desde el nivel macro sino en el nivel institucional intermedio y el espacio micro del ciudadano actuando colectivamente.

\subsection{La logística epistemológica}

Para atender esta problemática es necesario establecer desde qué concepto de exploración científica vamos a trabajar. Nuestra práctica se enraíza en el constructivismo social para el que:

Este enfoque resuelve el problema de la objetivación de la realidad incluyendo las representaciones (constructos, interpretaciones, descripciones) de diversos observadores. Aquello que convenimos como realidad aparentemente externa al sujeto y de la que podemos extraer, mediante nuestras observaciones, cierto orden, cierta estabilidad, requiere, como mínimo, la presencia de dos observadores en interacción que van creando y haciendo emerger un marco de referencia consistente (Romero Pérez, C. 2001) y (von Foerster, 1996).

Por lo tanto deducimos que

a.1. El conocimiento es un resultado colectivo.

a.2. El sujeto cognoscente, es decir, los involucrados en las explicaciones e interpretaciones, sus actores están implicados en el objeto y

a.3. la realidad no es externa al sujeto, sino que es una construcción a partir de las relaciones interactivas entre sujeto y entorno (Romero Pérez, C. 2009)

y consecuentemente:

(b) la realidad es co-construida mediante este juego interactivo.

Concluimos con Romero Pérez que los valores epistémicos que motivan esta reforma del pensamiento son, entre otros, los siguientes:

(a) conocer para hacer; es decir, combinar los conocimientos teóricos con los de acción;

(b) conocer para innovar; o lo que es igual, conocer para crear nuevos conocimientos, más allá del saber técnico-aplicacionista;

(c) conocer para repensar lo conocido o pensado; es decir, poner a prueba las categorías conceptuales con las que el científico o el tecnólogo trabajan para hacer inteligible o manipulable la realidad que se desea estudiar o sobre la que se desea intervenir. 


\section{2. ¿Cómo se gestiona conocimiento para la acción?}

Para responder a esta pregunta recurrimos a una corriente de pensamiento que se despliega con fuerza a partir de los años cincuenta del siglo pasado: el pensamiento sistémico. Frente a la crisis del pensamiento positivista y sus explicaciones monocausales o lineales se impone el desafío de obtener una comprensión integral de la realidad, basada en el conocer y gestionar la complejidad. El pensamiento sistémico reúne a un conjunto de pensadores, científicos y practicantes de la teoría de la acción, conscientes de la necesidad de construir y propagar nuevos enfoques, conceptos y herramientas metodológicas para entender mejor y de un modo interdisciplinario, la complejidad de la realidad actual, en orden a diseñar y co-construir un mundo más justo y sustentable (Senge, 1998).

Ludwig von Bertalanffy surge como el referente principal del paradigma sistémico, y es acompañado por relevantes figuras del pensamiento del siglo XX, como llya Prigogine, biólogo, De Geus, planificador y especialista organizacional y Jay Forrester, catedrático y precursor de los estudios sobre economía industrial tomado como un fenómeno sistémico (Von Bertalanffy, 1995).

Convergen en este trabajo en lo que hace al enfoque sistémico la visión sistémica o, lo que es lo mismo, relacional, procesual, no lineal o circular (recursividad) unida a los nuevos principios científicos que explican la emergencia de estructuras nuevas o más complejas (orden) a partir de lo imprevisible o aparentemente aleatorio (caos), y permiten explicar y comprender-aplicando esta nueva escala- aquellos fenómenos dinámicos (sistemas dinámicos) que ocurren en el mundo real natural o social. (Romero Pérez, Clara,2006)

A su vez, y con relación al concepto de complejidad en la terminología de la Sistémica, y a diferencia del vocabulario no especializado, complejidad no significa dificultad ni complicación, sino variedad. Un sistema complejo puede incluir tanto un número grande como uno pequeño de componentes, pero sus interrelaciones son de naturaleza sofisticada y no son lineales. La comunicación humana, por ejemplo, presenta este tipo de relaciones en las que todo está relacionado con todo en un entramado imposible de reducir a dos dimensiones (H-Coll, 2014).

Cuando nos referimos al sistema nacional mediante la articulación de un sistema de integración continental, atenderemos a los aportes de reconocidos intelectuales y científicos como Uri 
Bronfenbrenner, Pierre Bourdieu, Kurt Lewin y Ronald Burt así como la experiencia de referentes de procesos de integración regional, especialmente de la Unión Europea, entre los que contamos a Eduard Best y Les Metcalfe.

Nos detendremos ahora a describir algunos de los aportes de la teoría sistémica, pertinentes al contenido de este escrito.

Concepto de sistema: conjunto de partes de un todo, cuyo funcionamiento y resultados son superiores a la suma de las mismas. Aunque un tanto general, esta definición abre las puertas al fenómeno de la complejidad, sólo comprensible cuando se lo aborda desde diferentes perspectivas y con el aporte de estudios que nos acerquen al conocimiento más acabado de ese fenómeno complejo. Todo hecho humano es una realidad compleja: una pareja, una organización o la integración supranacional entre países.

\section{El enfoque sistémico}

Dentro de esta esfera de pensamiento se dan dos principios especialmente pertinentes para nuestro trabajo: sinergia y recursividad.

Sinergia se refiere a la acción de dos (o más) causas cuyo efecto es superior a la suma de los efectos individuales. La sinergia es la suma de energías individuales que se multiplica progresivamente, reflejándose sobre la totalidad del grupo.

Recursividad; es la propiedad que posee un sistema, o partes del mismo de reproducirse en una escala menor o mayor, manteniendo sus propiedades - o un conjunto de ellas- similares entre sí, con lo que cada una de una de esas partes refleja la totalidad. El hombre es un conjunto de células y el grupo es un conjunto de hombres. Podemos establecer una recursividad célula - hombre - grupo. Pensemos en la empresa como una totalidad y pensemos en solo dos aspectos de ella: dirección y producción. Ambos niveles se reproducen, a cualquier nivel - subsistema dentro de la empresa; gerencia, jefatura departamental, división, etc. A este elemento que se reproduce en cada nivel de una empresa o una organización se lo denomina fractal. Es así como es posible considerar a los equipos de trabajo como un fractal organizacional que se reproduce en su sistema mayor/empresa. Michel Henric Coll amplía su enfoque de esta manera: 
He imaginado un modelo en el que toda la empresa es vista como un equipo, cualquiera que sea el nivel de aproximación que adoptemos. Eso es: un equipo formado por equipos en diferentes niveles de profundidad. Tanto vista en su globalidad como en cualquier nivel de su organización, la empresa existe y funciona como un equipo. Debido al isomorfismo, es suficiente comprender y saber hacer funcionar el elemento 'equipo para que funcione toda la organización, y como todo es homogéneo, no se producen rechazos ni discordancias. Homogéneo, simple y generador de complejidad. He llamado a esta organización, organización en equipos fractales, o más simplemente: equipos fractales (fractal teams). (Michel Henric Coll, 2014, p.1)

De igual modo podemos ampliar el rango de ejemplos de organizaciones fractales que conllevan por su naturaleza dispositivos colaborativos.

Sólo para mencionar un grupo de ellos, pertinentes a esta problemática - y que serán identificados en los casos analizados-, mencionamos el diseño de una visión de futuro, trabajo en equipos, redes de aprendizaje, métodos de gestión del conocimiento y sistemas digitales de comunicación entre personas y grupos.

\section{Ejemplo de fractales.}

Como ejemplos de un fractal aquí mostramos dos figuras de fractales que componen un triángulo, sólo difieren en su grado de complejidad. La figura 1 muestra 2 modos de triángulos; el más amplio e inclusivo que contiene a 4 cuatro triángulos, uno de ellos invertido.

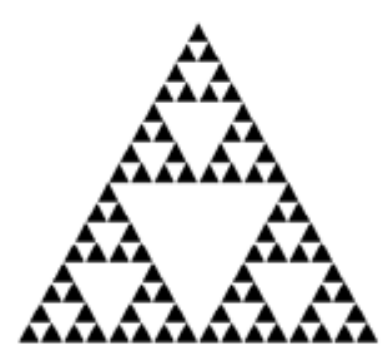

Figura 1

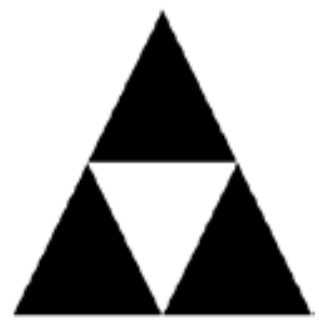

Figura 2

La segunda figura muestra 5 tamaños, donde de mayor a menor se incluyen todos los "niveles". En el ejemplo de la empresa -mencionado antes-, la misma estaría representada por el triángulo mayor, el triangulo invertido blanco correspondería a la gerencia general, el tercer tamaño es el de las gerencias funcionales, el cuarto tipo a divisiones y los triángulos más pequeños a departamentos y/o proyectos. 


\section{Las redes como fractales}

Las redes como fractales que sostienen una construcción institucional.

Lo que sostenemos en este trabajo es que la red organizacional constituye uno de los fractales principales para articular un espacio de integración. Una característica distintiva de los procesos organizacionales en todos los niveles sistémicos es la importancia central de las redes organizacionales, herramienta de comunicación y acción que para muchos identifica esta etapa de desarrollo global.

Veremos aquí, como ya lo hemos citado, las redes de aprendizaje como una estructura central, y específicamente nos referiremos al tipo de red flexible, que se sostiene en el concepto de "organización que aprende", y recibe el nombre de red de vinculación y aprendizaje (Senge, 1998).

Definimos entonces a la red como un entramado de vínculos entre dos o más actores, cuya estructura facilita y promueve la auto-organización de sus integrantes, la conciencia de esas conexiones, y su activación a través de compromisos que generan resultados para el conjunto y para cada integrante.

\section{Cómo operan las Redes de Aprendizaje}

- A través de proyectos, cuya iniciativa puede residir en una persona o en algún sistema colectivo (grupo, empresa, organización)

- El proyecto debe integrar una visión común de lo que se desea obtener

- Esta visión debe traducirse en resultados medibles (cantidad, calidad y tiempos)

- Los resultados en actividades, y estas, en roles, Integrados en procesos (flujos de trabajo)

- Para los cuales se asumen las responsabilidades por cada actividad que deben cumplir con ellas.

- La actividad de la red se evalúa a través de indicadores, en tanto instrumentos de control y soportes del proceso de auto-organización de la misma. En otras palabras, la red puede autocorregirse si los indicadores de desempeño establecidos no se cumplen

\section{valor de los contextos.}

Finalmente, una estructura flexible en sistemas complejos sólo puede sostenerse a través de una inserción sinérgica con sus contextos organizacionales. Distinguimos aquí cinco tipos de contextos, que se caracterizan por favorecer o neutralizar esfuerzos asociativos dependiendo tanto del análisis que se haga de los mismos como de la intencionalidad institucional de obtener resultados esperados. 
Naturalmente hay un camino analítico para recorrer los contextos que hace posible las acciones colaborativas, que haremos a continuación.

\section{El contexto 1: la actitud y estrategias del ciudadano.}

Para establecer una lógica desde el origen, y tratándose de trabajar colaborativamente, con iniciativas de cambio (social/societario) instituyentes debemos empezar desde la persona, viviendo y actuando con otros. Esta persona vive en su habitus, definido como "un sistema de disposiciones adquiridas por medio del aprendizaje implícito o explícito que funciona como un sistema de esquemas generadores, genera estrategias que pueden estar objetivamente conformes con los objetivos de sus autores sin haber sido expresamente con este fin" (Bordieu, 2003, p.125). Es lo que denominamos el contexto de base. Ya en esta etapa deben desarrollarse los genes colaborativos, por actitud heredada o rutinas aprendidas. Esto habilita a comportamientos abiertos al compartir y cooperar de modo natural.

Contexto 2 - En tanto que ciudadanos actuamos en tres espacios: estado, actividad productiva -"privado"- y sociedad civil, con sus asociaciones (Bobbio, 2006). El corolario: habrá una ciudadanía más rica en tanto los tres sectores se entretejan de un modo sinérgico.

Contexto 3 - El continuo local-internacional, que a partir de los 80 se aprecia con claridad en la internacionalización (globalización) de las TICS y el despliegue de la galaxia internet (Castells, 2001). Incluye a territorios comunales, subnacionales, nacionales e internacionales, los que interactúan de modo permanente.

Contexto 4 - El espacio productivo y socio comunitario -de las cadenas de valor, tantas como productos y servicios existen- con la convergencia de los procesos de gestión de conocimiento científico y tecnológico que se generan en su interior y de las instituciones de educación y asistencia técnica especializadas.

Contexto 5 - El contexto general como un sistema integrador. Es transversal a los anteriores, en el que cada vínculo se sitúa en una de las tres capas, macro, meso y micro, de las cuales la capa meso contiene poder generativo, con agentes claros de transformación social. La sociedad como un ambiente ecológico, al interior de la cual distinguimos tres sistemas que atraviesan los 4 contextos anteriores: micro, meso, y macro. Vamos a explayarnos más ampliamente sobre este tema a continuación. 
valor diferencial del espacio mesoinstitucional.

Definimos el espacio Meso Institucional como el conjunto de actores, reglas, instituciones $y$ organizaciones públicas, privadas y de la sociedad civil, que procesan-reciben, adaptan, reformulan, completan y/o rechazan- las actividades entre el espacio micro y el macro.

Por lo tanto este dominio, abarca:

1. toda la gama de actores y recursos de los distintos "colectivos ciudadanos sectoriales" del sistema socioproductivo, científico tecnológico

2. Ios funcionarios de los estamentos medios de la esfera pública estatal

3. Ios profesionales, gerentes de la esfera privada, y dirigentes de empresarios y trabajadores

4. los investigadores, científicos y tecnólogos de las áreas de ciencia y tecnología

5. el conjunto de artistas, artesanos y docentes de la cultura, el arte, la educación

Estos actores y posiciones constituyen la base a ser analizada junto con las redes sociales $y$ organizacionales en las que están articulados.

A su vez cuentan con un dinamismo propio que Bronfenbrenner describe del siguiente modo:

El potencial evolutivo de los entornos de un mesosistema se ve incrementado si las demandas de roles de los diferentes entornos son compatibles, y si los roles, las actividades y las díadas en las que participa la persona/ institución en desarrollo estimulan la aparición de la confianza mutua, una orientación positiva, el consenso de metas entre entornos, y un creciente equilibrio de poderes a favor de la persona/ institución en desarrollo (Bronfenbrenner, 1987, p.28).

\section{Los dispositivos del modelo.}

Para armonizar los componentes sistémicos y articularlos con los contextos antes mencionados son necesarios dispositivos ${ }^{2}$ que lo hagan posible. Para definir este concepto recurrimos al diccionario de la Real Academia Española (2014): un dispositivo es un mecanismo o artificio dispuesto para producir una acción prevista. Al tomar como objeto de este modelo proyectos estructurados "desde abajo" es necesario prever - e instrumentar- sus alineamientos con (a) espacios institucionales públicos, privados y de la sociedad civil y (b) de los contextos locales, nacional y macroregional.

En particular, y dado que nuestro foco es la acción colaborativa, recurrimos a los drivers o dispositivos de acople del sistema definidos como mecanismos que garantizan información completa,

\footnotetext{
${ }^{2}$ Usamos también los conceptos de drivers o comandos, tomados del lenguaje computacional, que asimilamos al
} significado de dispositivo. 
comunicación abierta, construcción de confianza y acción colaborativa sustentable. Y en el campo de los valores, en el espacio del desarrollo humano sustentable, se trata de generar confianza mutua, una orientación positiva, el consenso de metas entre entornos, y un creciente equilibrio de poderes a favor de la persona en desarrollo.

\section{Metodología.}

La metodología elegida es el estudio colectivo de casos, de interés instrumental, lo que nos permitirá reflexionar y desplegar la característica más distintiva de la indagación cualitativa, cual es la interpretación (Stake, 1997).

En esta modalidad de estudio es de importancia central la función interpretativa constante del investigador, que participa de la misma dimensión semántica que la búsqueda de sentido sensemaking- dado un conjunto sistémico de acciones (Stake, 1997, p.47). Esta modalidad metodológica se distingue por su acento en el trato holístico de los fenómenos. "Ya he señalado que la epistemología del investigador cualitativo es existencial (no determinista) y constructivista" (Schwandt, 1994). En los estudios cualitativos se esperan "descripciones abiertas", "comprensión mediante la experiencia" y "realidades múltiples".

El insumo principal de este artículo es el trabajo de recolección de datos para la tesis doctoral del autor (2014) con los casos estudiados en dos territorios nacionales: Brasil, con el testimonio de los actores de un continuo institucional que incluye el Estado de Minas Gerais, el Instituto Nacional de Telecomunicaciones - INATEL, y el Municipio de Santa Rita de Sapucaí; y en los Estados Unidos de Norteamérica el Sistema Universitario y el Científico Tecnológico del Estado de Georgia. En el texto de referencia hemos fundado la validez de las comparaciones entre los casos, que nos permiten inferir conclusiones de equivalencia entre ellos.

La razón por la que se eligieron estriba en que ambos representan una experiencia consolidada en las últimas décadas con una riqueza inusual en cuanto a los contenidos, evaluados por tres criterios: diversidad institucional de cada caso, elevada interacción con los contextos y potencial de colaboración entre organizaciones fractales.

En función de estos criterios ampliamos la información sobre cada territorio.

- Para el caso de Minas Gerais, elegimos los procesos fundacionales del distrito industrial de 
Santa Rita, INATEL la Escuela Técnica de Electrónica (ETÉ), y la cobertura obtenida por políticas estaduales del Gobierno y Estado de Minas Gerais y capacidades colaborativas del propio territorio.

El período estudiado se extiende desde fines de los años '50 hasta la actualidad. En él se verifica:

- a. Diversidad institucional: la generación de centros de excelencia en el plano educativo y tecnológico en el municipio de Santa Rita, al sur del estado de Minas Gerais, equidistante a 250 kms. de los 2 núcleos urbanos y tecnológicos más importantes de Brasil; San Pablo y Río de Janeiro. El naciente distrito industrial queda conformado básicamente por la Escuela Técnica de Electrónica de Santa Rita, el INATEL, Instituto Nacional de Telecomunicaciones de Brasil, la Facultad de Informática -FAI - el Sindicato de Empresas del Valle de la Electrónica - SINDVEL; 140 firmas a la fecha.

- b. elevada interacción con los contextos: la voluntad emprendedora de esa comunidad, provoca el apoyo del Estado de Minas y su Secretaría de Ciencia y Tecnología - SECTES - nivel mesoinstitucional; y en varias ocasiones de los gobiernos nacionales de Brasil, nivel macro, induciendo un enriquecimiento institucional en ambos espacios instituyentes.

- c. potencial de colaboración entre organizaciones fractales: tomado el conjunto de actores mencionados en el punto anterior como una estructura sistémica colectiva que gestiona conocimiento, quedan establecidas las bases para un juego multiactoral e interdisciplinario en una espiral ascendente de nuevo conocimiento social y tecnológico, seguida por innovaciones socioproductivas y desarrollo integral del territorio. En 2011, INATEL, junto con la sociedad de agentes ya referidos, fundan el Parque Tecnológico para gestionar conocimiento del territorio, utilizando los fractales expuestos más arriba, entre otros: redes de aprendizaje y redes densas con trabajo en equipos.

En el Estado de Georgia, EUA, seleccionamos la estructura del Sistema Universitario de Georgia SUG - , conformado por las 34 universidades públicas del Estado, con un total de 240.000 alumnos y el proyecto científico de tecnología de banda ancha, Yamacraw,

- a. Diversidad institucional. Para tener una idea aproximada sobre este punto, dos de las 34 universidades del sistema GSU y Ga Tech, cuentan con 114 Centros de investigación, y muchos de ellos integran consorcios público-privados nacionales e internacionales. Además, el Estado de Georgia cuenta con 54 delegaciones Comerciales en el exterior, incluyendo una 
representación ante la UE en Bruselas.

- b. elevada interacción con los contextos: EI SUG se crea en el año 1930, con compromisos iniciados 8 años antes y refrendados en actas acuerdo. Se inicia como una iniciativa de ciudadanos y se transforma en una institución de carácter público, federalizada, con un Consejo de Regentes que representan a los 18 departamentos counties- del Estado.

- c. potencial de colaboración entre organizaciones fractales: extensa mundialización de relaciones tecnológicas, de educación superior, público-privadas en los 5 continentes.

\section{Resultados:}

Para tener una descripción significativa aportaremos la dimensión dinámico-histórica de los hechos, lo que nos permitirá identificar las fases temporales de cada dispositivo, así como su inserción en uno o varios contextos.

Pasamos ahora al análisis descriptivo y asignación de sentido a los ítems a tratar, tomando como base de diferenciación los tres sistemas del ambiente ecológico y los grados de alineación al interior de cada uno y entre ellos.

\section{Sistema micro}

En el nivel micro-organizacional es fundamental la actitud de los individuos o de la unidad base según se trate de familia, grupo de amigos o alumnos de una clase. En nuestra perspectiva "Ios mecanismos para producir una acción prevista" en un modo colaborativo y/o asociativo requieren de una actitud emprendedora. El análisis que proponemos supone analizar actores individuales e institucionales dotados de una capacidad proactiva hacia el cambio a niveles de mayor bienestar y desarrollo colectivo.

Fase 1: Dispositivos de apertura a la diversidad, proveen adaptación o creación de contextos.

La apertura "emerge cuando dos o más individuos están dispuestos a suspender la certidumbre en presencia del otro. Están dispuestos a compartir los pensamientos y a dejarse influir por el otro." (Senge, 1998, p.354)

Los indicadores principales son las redes densas, redes de confianza que porta cada actor y las redes extensas, definidas como cadenas de contactos de conocidos, del modo en que aparecen las redes sociales internetianas, o simplemente, en una búsqueda por el espacio virtual, por la que se 
descubren contactos desconocidos hasta el momento, y se los incorpora como enlaces útiles.

* Las redes densas se generan por doquier, cuando existe un verdadero clima de producción y creatividad; como ejemplos tenemos en Brasil, al inicio de INATEL, década del '60, que contó con el apoyo de una densa red interinstitucional, la Escuela Técnica Electrónica, grupo de amigos del intendente del municipio, grupo de profesores, la casa de la Amistad, las relaciones de respeto y trabajo con funcionarios y directivos de las empresas de telecomunicaciones, y funcionarios nacionales en Río de Janeiro y en la legislatura estadual de Minas Gerais y nacional de Brasil.

En Georgia, el cuerpo de Regentes, con inicio en 1930, la estructura que gerencia el Sistema Universitario de Georgia y sus 34 universidades tienen la misión de distribuir recursos, administrar infraestructura y la currícula de 240.000 alumnos. Con 18 miembros, se reúne una vez al mes para tratar una agenda nutrida representando a todos los distritos municipales de Georgia. El acta de cada reunión está disponible en la semana siguiente por internet.

* Redes extensas son las que se construyen con conocidos de conocidos; esto ocurre de modo sistemático cuando iniciamos una búsqueda en internet o participamos de las redes sociales. De un modo más elaborado lo realizan quienes trabajan en el observatorio radar del Polo Tecnológico de Santa Rita, para obtener información clave de puntos lejanos, en el escenario mundo.

Los dos recursos mencionados, interactuando uno con otro, constituyen el inicio necesario para identificar analíticamente un escenario con potencial de acción colaborativa lasociativa y actuar sobre él.

*Actitudes y acciones de liderazgo: Así la gestión de líderes fundadores locales,- otro fractal centralexpresan conductas de mente abierta, foco estratégico y alineamientos colectivos.

Los propios actores hablan así de los emprendedores institucionales, co-fundadores de INATEL;

En marzo de 1964 los profesores de la Universidad de Itajubá; - José Nogueira Leite, Fredmark Leao y Fernando Costanti, deciden viajar a Santa Rita de Sapucaí, ante la imposibilidad de llevar adelante su iniciativa, por la caída del gobierno del Jango Goulart, y la falta de fondos con los que iban a construir la Facultad de Electrónica en Itajaí -distante 200 kms. de Santa Rita. (INATEL, 2002).

A los que se suman con sus visiones fundacionales la Señora Moreira en la creación de la Escuela Ténica Electrónica e INATEL; y el prefecto de Santa Rita, Paulo Federico de Toledo, con su persistente acción sobre los empresarios.

El driver en este espacio micro se completa con: 
- Enlaces redundantes para acceder a personas en cada contexto; en oposición a estructuras cerradas con accesibilidad limitada por reglas tácitas o informales.

- Y espacios abiertos a nuevos actores y temas emergentes, en toda instancia de programación, cadena de decisiones y cursos de acción.

Todos los mecanismos mencionados - que constituyen al mismo tiempo recursos-, se potencian cuando conforman redes de vinculación y aprendizaje (RedesVyA).

El Polo Tecnológico Electrónico de Santa Rita, con sede en INATEL, cumple con este rol, en tanto que central de gestión de conocimiento tecnológico local/ nacional/ internacional. Este Polo PE-ET está definido por sus actores como

una plataforma de programas, proyectos e iniciativas de base científica y tecnológica. Como tal, se propone promover la organización y el fortalecimiento de las estructuras de gerencia y de transferencia de conocimiento del sector, en el ámbito nacional. Constituido como esfuerzo pionero en la enseñanza técnica de Electrónica en el país y América Latina e internacional (INATEL, 2009, p. 3).

Ampliando el lente de observación vemos que el conjunto de "los actores institucionales con centro en Santa Rita: Inatel, Facultad de Administración e, Sindvel, Municipio, actúan como una Red de Vinculación y Aprendizaje, sumando nuevas instituciones /Red: Secretaría de Ciencia y Técnica del municipio y la formalización legal del Polo Electro Electrónico Tecnológico y las Incubadoras de empresas de INATEL y Municipio. Como contexto geográfico meso rodea a este espacio el estado de Minas Gerais y en él la SECTES-Secretaria de Ciencia, Técnica y Educación Superior- ; con sus 298 instituciones, incluidos los programas de CyT, Educación Superior y activación socio-productiva."

Sistema meso-organizacional

Fase 2: Los dispositivos mencionados -micro-interactúan con los propios de este sistema.

Drivers del proceso institucional de apertura

La función de este driver es traducir las estrategias a proyectos, y estos a procesos actuales y a emergentes, consensuados colectivamente y con sus flujos de trabajo integrados.

A estos componentes organizacionales deben agregarse los espacios de roles, propios de las redes: exploradores, articuladores, mentores/ padrinos, y coordinadores.

Estos drivers cuentan como insumo principal a los análisis de campo- tecnológico, productivo, educacional- $y$ de contextos, con especificación de actores dominantes, actores hegemónicos, 
áreas de colaboración y de ruptura o conflicto, tal como se ejemplifica en el Plan Director para Minas Gerais y los planes estratégicos de la SeCTES, Minas Gerais, que explicitaremos de inmediato

Fase 3: Completada la activación de fractales y drivers anteriores corresponde proceder con esta fase.

Drivers de previsión y diseño de alianzas para conseguir escala, diversidad o resultados no accesibles para iniciativas aisladas. Este dispositivo requiere de equipos multidisciplinarios que trabajen interdisciplinariamente, y del análisis de escenarios realistas y estrategias acordes. Un ejemplo claro y complejo es la iniciativa Yamacraw, en el estado de Georgia, EUA; que exponemos en detalle al final de este parágrafo.

Dispositivos de Negociación / articulaciones para alineamientos posibles entre los actores: el que básicamente tiene a los actores meso como agentes fundamentales.

Un subtipo de dicha relación: Estrategias alineadas de Provincias con sus municipios, de estos entre sí, y con contrapartes internacionales en las duplas Estado-Sociedad Civil y Estado - Sector socioproductivo, Tecnológico y Enseñanza Técnica y Superior.

Ejemplo completo: La sinergia de las políticas macro y estrategias meso del Estado de Minas Gerais, 2003/2014, las propias del SECTES y potencial de alineamientos con las iniciativas innovadoras de los municipios e instituciones de Educación Superior del estado, tal como ocurre de un modo paradigmático - aunque no único- con el continuo municipio de Santa Rita- INATEL, Polo Tecnológico e instituciones educativas y científicas de este territorio de 40.000 personas. Estas duplas se comunican a través de actores espejo en cada espacio: por ejemplo, gerentes y funcionarios de la sociedad civil, con pares de la Administración Pública local o nacional y con los niveles medios de empresa. $^{3}$

Drivers de aprovechamiento de Áreas de oportunidad /crecimiento.

Identificables por análisis de Fuerzas/ Oportunidades/Debilidades/Amenazas de cada proyecto, desplegados con métodos de prospectiva, de escenarios futuros en los que se analizan y prevén potenciales alineamientos de actores con oportunidades para el proyecto, a la vez que se atienden

\footnotetext{
${ }^{3}$ Podríamos decir, "a la japonesa", en la que los actores medios son disparadores de innovación hacia los mandos superiores y después a hacia los niveles operativos. Ver Nonaka, (1999); Capítulo 3" El modelo "centro-arriba-abajo" un proceso de administración para la creación del Conocimiento" en "La organización creadora de conocimiento", pp.137176.
} 
las amenazas y debilidades de la acción prevista en este último.

Incluye los siguientes recursos

- Drivers para Áreas de transición: integradas por agentes institucionales que por su rol natural o por compromiso autónomo asumen, durante un período, la función de incubadora de una actividad, proceso o resultado completo del proyecto.

- Dispositivos de "saber negociar" entre actores de diverso nivel, sector y jerarquía.

Fase 4. Es la extensión al continuo local -internacional.

Driver base: la cooperación técnica internacional. Al amplísimo campo de cooperación y alianzas entre espacios e instituciones locales entre sí -ciudades gemelas, acuerdos de complementación entre empresas, provincias y jurisdicciones nacionales, debe agregarse el campo- dispositivo de la cooperación técnica internacional, como un cauce de metodologías que normalmente implican acciones colaborativas o de aprendizaje colaborativo, instrumento disparador de procesos innovadores en territorios receptores de la misma.

- Esto se visibiliza en el proceso de afianzamiento de INATEL y de la comuna de Santa Rita, las que durante los años 1994 y 2005 mantuvieron un intercambio amplia-mente aleccionador con comunas y cámaras y empresas alemanas a través de la GTZ, el organismo de cooperación internacional de Alemania.

Dispositivos de interoperabilidad e interusabilidad. En una línea transversal a los drivers mencionados deben sumárseles los mecanismos que actúan, a la vez, como condiciones de:

a. interoperabilidad, que los equipamientos y los programas sean manejables por el ciudadano en cualquier territorio (municipio, país, continente) en el que se encuentre, con procedimientos también fáciles de operar.

b. interusabilidad de los textos, y documentos: que el ciudadano acceda a información transparente, pública, fácil de ubicar. La interoperabilidad de los instrumentos de acceso debe complementarse con el principio de interusabilidad de todos los ciudadanos. Dicho más simple, que los códigos -normalmente en lenguaje digital - tanto como los textos sean operables y usables por el ciudadano.

En el espacio de la articulación meso-macro, 
Un Plan Estratégico es en si mismo un fractal - componente reproducible en cada sistema- y driver -instrumento articulador- de la mayor importancia, cuando tratamos el desarrollo de iniciativas macromeso; es decir con formulación colaborativa en la esfera del gobierno y con instrumentos precisos en el nivel ejecutivo meso; tal como es el Plan Director 2009-2013.

Elegimos como introducción al tema el ejemplo del gobierno de Minas Gerais.

Redes para la gobernabilidad: en la nueva etapa del Gobernador Antonio Anastasia -20102014- los resultados se reorientan hacia un estado con base en redes, tal como reza la consigna del título de su Plan Estratégico, en cuyo prólogo se describe de la siguiente manera: "Nuestro programa se propone estructurar una nueva forma de gobernar que tenga en cuenta el horizonte que Brasil debe enfrentar en los próximos años, a través de la formación y estructuración de redes de desarrollo integrado para alcanzar el binomio de nuestra administración: vida y renta, calidad de vida y de trabajo de las personas y nuestra perspectiva de desarrollos (SECTES, 2009).

Se exigen también nuevas formas de gobernanza a través de redes asociativas con la sociedad civil, poderes políticos nacionales y locales, empresarios y entidades de la sociedad civil clase. Otro proceso fundamental será buscar mecanismos que propicien la transversalidad sectorial, en el sentido de desarrollar soluciones que integren las diversas formas de conocimiento y organizaciones disponibles en Minas."

La expresión de este plan estratégico se ve en los Planes y estrategias del Plan Director 20092013, donde se despliegan los "saber cómo"' de actores y cursos de acción relevantes. En 2007 la SECTES elaboró mapas estratégicos para las políticas de Ciencia, Tecnología, Innovación (CTI) y Enseñanza superior (ES) con un horizonte de 5 años. Aquí resumimos las partes relevantes al objeto de nuestro trabajo. Todas ellas cumplen con el principio de recursividad, pudiendo reproducirse en cada nivel del sistema total.

Misión, visión y valores. (SECTES, 200p9)

- Misión: Promover la ciencia, la tecnología, la innovación y la enseñanza superior para el desarrollo sustentable y la mejora de la calidad de vida en Minas Gerais.

- Visión: Ser referente nacional en la formulación y en la implementación de políticas públicas que aseguren el desarrollo científico y tecnológico, la innovación y la enseñanza superior

- Valores: compromiso, cooperación, creatividad, ética, excelencia, respeto y verdad.

Cuatro perspectivas convergentes. A continuación sintetizamos las cuatro perspectivas, -

${ }^{4}$ Registro de notas de campo del autor. 
aprendizaje, procesos, socios y Minas Gerais- en que se escalona el Plan Director de MG, dando comienzo con objetivos en el espacio de aprendizaje, es decir, implican un aprendizaje permanente y por tanto, mejora permanente.

\section{De aprendizaje ${ }^{5}$}

- Actuar con foco en competencias estratégicas. Lo que implica las siguientes competencias gerenciales: orientación para resultados, visión sistémica, co-participar informaciones y conocimientos, liderazgo de equipos, gestión de personas, competencia técnica y capacidad innovadora.

- Consolidar el modelo de SECTES, “de Excelencia de Gestión”, constituido por 8 criterios: liderazgo, estrategias y planes basados en estudios de mercado y del macro ambiente, segmentación y estrechamiento del vínculo con los clientes, cumplimiento de la responsabilidad medio-ambiental, gestión estratégica de información y conocimiento, gestión de personas, mejora de los procesos organizacionales y resultados, esto es análisis de resultados en los 7 puntos anteriores.

- Perfeccionar la gestión de la información y el conocimiento ${ }^{6}$

- Así, "promover la gestión y la utilización de información posibilita compartir las mejores prácticas organizacionales, la divulgación corporativa - organizacional del conocimiento y establecer ventajas competitivas".

2. Perspectiva de procesos. Estamos aquí ante procesos meso estaduales, y debe observarse el énfasis en preservar el capital intelectual - retención de talentos -, las normas "el ordenamiento jurídico que respete las dinámicas de CTi y Educación Superior" y la prospectiva para agudizar la vista en los escenarios futuros:

- Fortalecer la investigación científica y tecnológica, los Centros de Excelencia y la retención de talentos.

- Estimular la creación y fortalecimiento de cursos de base tecnológica y profesionalizante.

- Ampliar la oferta de Enseñanza Superior (ES) por medio de Enseñanza a Distancia.

- Buscar ordenamiento jurídico y regulatorio compatible con las características y dinámicas del área de CTI y ES y producir análisis prospectivos para inducir acciones estratégicas y subsidiar políticas públicas en áreas CTI y ES.

5 Mantenemos aquí el número original de objetivo, tal como figura en el Plan Director.

6 Autor (2014) Observar la implicación de ambos procesos: generar información completa es una etapa principal de la gestión del conocimiento 
- Establecer directrices para la Política de Comunicaciones de la SECTES y popularización de CTI.

3. Perspectiva socios o, en nuestro concepto, driver de articulación del capital social-institucional en el nivel meso, articulado con el nivel macro estadual.

- Apoyar los programas y agentes de Tecnología Industrial Básica;

- Revitalizar la capacidad de gestión y articulación para impulsar el Sistema Estadual de CTI y Enseñanza Superior;

- Acelerar y valorizar el proceso y la cultura de innovación. Objetivo: consolidar el Sistema Mineiro de Innovación/ SIMI, como referencia en gobernabilidad de procesos de innovación.

- Perfeccionar la interacción entre Instituciones de Educación superior, Institutos CyT y sector productivo y

- Consolidar centros de I\&D e Innovación.

- Desarrollo regional y sectorial, por medio de Alianzas Productivas Locales y Polos de Excelencia e Innovación, y estimular emprendimientos de base tecnológica.

4. - Perspectiva Minas Gerais o Dispositivo de articulación Macroestadual (Minas Gerais) con macronacional (Brasil)

- Transformar Conocimientos en negocios elevando productividad y competitividad. Posicionar a MG como líder de Economía del conocimiento en Brasil

- Consolidar en la sociedad la percepción de CTI+ES como área estratégica y alinear los indicadores del sistema de CTI+ES con el Programa Mineiro de Desarrollo Integrado. (SEXCTES, 2009, pp. 35-40)

\section{Corolario sobre las cuatros políticas:}

\footnotetext{
- Se trata de una suerte de "Manual sobre dispositivos de una organización flexible", especialmente, cuando se observa la imbricación intrínseca de las cuatro políticas.

- Se infiere la importancia de aumentar densidad tecnológica y de conocimientos en el diseño de productos, servicios y organizaciones.
} 


\section{La Iniciativa Yamacraw -Estado de Georgia-EUA}

Un caso completo de los alcances, diversidad y estructura que debe sostener un sistema con sus fractales sistémicos y drivers colaborativos es la iniciativa Yamacraw. En el mes de Enero de 1999 el Gobernador de Georgia Roy Barnes anuncia el inicio de la iniciativa Yamacraw, de 5 a 7 años de duración y una inversión de u\$s100.000.000. Ha sido una iniciativa piloto que reúne casi todos los atributos de un proyecto exitoso,

El Dr. Raymond Greenlaw, impulsor y coordinador de esta iniciativa hasta el año 2005, la describe de esta manera: "Yamacraw es una iniciativa de desarrollo económico para hacer de Georgia un líder mundial en el sistema de comunicaciones de banda ancha, alta velocidad, dispositivos y chips - la infraestructura de última generación - creando en Georgia puestos de trabajo de alto valor y muchos más puestos de trabajo en las actividades de soporte y cadena de proveedores."7

Es una iniciativa federal, dirigida desde la Universidad de Armstrong Atlantic, de la ciudad de Savannah a 400 kms. de Atlanta. También, y de modo principal, es un proyecto multi-institucional, que ha comprometido a varias universidades, municipios, centros de investigación - uno de ellos, el GCATT la ha integrado a su estructura- empresas privadas, fondos de inversión. Ha tenido múltiples áreas de impacto: en la Educación superior, en la estructura productiva, en el sector científico, en el impulso de herramientas financieras y, finalmente, en la identidad del Estado.

Estos logros los resumimos aquí, tomando como base la exposición del Dr. R. Greenlaw, insertando nuestras notas, mostrando como la diversidad en cada caso aporta a un patrimonio común de prácticas socio-productivas, políticas y culturalmente alineadas.

Fase 1: Inicio: Contexto histórico político. El programa se origina en conversaciones entre investigadores y contactos en empresas.

Metas Gerenciales con indicadores.

- Trabajos: Crear 2000 nuevos trabajos de alta tecnología en Georgia

- Centro de investigación: Centro de Georgia de Tecnologías Avanzadas en Telecomunicaciones. (GCATT, en inglés)

- Invertir: U\$S 5.000.000 anualmente en investigación, mediante fondos semilla.

- Atraer al menos 10 empresas para establecer centros de diseño de banda ancha en Georgia.

7 Los datos de este parágrafo sintetizan la presentación del Dr. Raymond Greenlaw, Coordinador Regional de Yamacraw, Decano, Escuela de Computación, Universidad Estadual Armstrong Atlantic. Tomado de internet en Marzo 2012. Recuperado: 2014, 10 de febrero. Disponible en: http://susancontreras.files.wordpress.com/2011/08/yamacrawlarge-brochure.pdf 
Fase 2- de Ejecución de los proyectos:

Co-Participaron los Comités de Comercialización, Marketing, Recursos Humanos y Actualización de Curriculums; acompañados por Marketing del territorio local/Estadual, haciendo conocer los actores de esta notable gestión del conocimiento colectiva e institucional. Premiada por el Consejo de la Competitividad como Líder Nacional en Iniciativas de Políticas Gubernamentales, se propone generar el reconocimiento de Georgia como un destino global de tecnología de banda ancha.

Desde el punto de vista estructural, este resultado es equivalente al APL del valle de la Electrónica, Minas Gerais, Brasil:

\section{Fase 3: Resultados:}

En clave de contexto 1;

Líderes: incorporación de Expertos Eminentes el Estado de Georgia. Dr. Joy Laskar; en Hardware: Diseño de Semiconductores y de dispositivos opto-electrónicos y diseño de accesos a Banda Ancha: y John A. Copeland, profesor, Facultad de Energía Eléctrica y computacional, GCATT/ la Alianza de Investigación de Georgia, experto eminente en transferencia de Tecnología.

- En Software Embebido: Dr. Vijay Madisetti ; en Prototipos de Sistemas y Dr. Nikil Jayant, profesor, experto eminente en tecnología inalámbrica, de la Facultad de Energía Eléctrica y computacional, GCATT y la Alianza de Investigación de Georgia, a cargo de prototipos de redes de ópticas de alta velocidad e inalámbricas.

- Fractal organizacional micro (profesores) a nivel meso en el área de Extensión Universitaria: Ampliación de la educación e investigación a las universidades estaduales a través de contratos a 72 profesores universitarios y constitución de equipos de trabajo inter y multidisciplinarios, y equipos de investigación multiuniversidad de 250 integrantes, colaboradores de "clase mundial".

- Más del doble de graduados especializados -826- en tres años. Sobre 400 como promedio anual en el área de las TICs /computación.

- Fortalecimiento del sistema educativo superior, federal e institucional.

Contratar 85 graduados de Ciencias de la Computación en el Sistema Universitario de Georgia y desarrollar y mantener la currícula al estado del arte.

Universidades Participantes; Integraron el consorcio ocho universidades del Estado. Obsérvese el 
criterio de distribución federal al interior del Estado, las universidades foco del proyecto no pertenecen a la capital del estado;

- Curriculum focalizado en Yamacraw: Armstrong Atlantic State University, Georgia Southern University, Savannah State University

- Con Investigaciones con curriculum focalizado: Georgia Tech, Southern Polytechnic State University, Georgia State University, University of Georgia, y Kennesaw State University.

En clave de contexto 2: las normas portan el criterio de sustentabilidad a fin de sostener la inclusión de actores e impedir acciones mono / oligopólicas. Por este requisito, las empresas deben tener base en Georgia, con desarrollo desde lo local, afirmando la capacidad e identidad del territorio. Debe sumarse a este la regla de "3 x 1": por cada dólar invertido por el Programa (universidad) el sector privado debe aportar tres. En lo que hace a la propiedad de los activos solo puede ser adquirido hasta un máximo de 49\% de propiedad de una compañía y no más de U\$S 500.000 puede ser invertido en la ronda semilla. Esto es para evitar el monopolio, o la cooptación del proyecto.

En clave de contexto 3: aumentar la visibilidad global de Georgia -- como destino tecnológico, y Yamacraw es difundida como prototipo probado para las iniciativas futuras de desarrollo económico de Georgia.

- Dar visibilidad y transparencia al programa. Aplicar una política completa de marketing y ventas, y asesorar en relaciones públicas y contrataciones corporativas. Generar el sitio web www.yamacraw.org - hasta que se incorporó al Centro de Avanzada en Telecomunicaciones y Tecnología de Georgia / GCATT. Finalmente, sumar a profesionales "repatriados" y confeccionar una base de datos dinámica de Curriculum Vitae.

En clave de contexto 4: Impacto en la industria de Georgia, con alcance internacional: Constituir un nuevo racimo (cluster) tecnológico, con la concurrencia de centros de diseños telecomunicacionales líderes: proveedor líder de soluciones de redes ópticas, firmas de diseño de semiconductores y conglomerado de telecomunicaciones en EE.UU. con socios europeos. A este logro se suman los compromisos de trabajo y creación de posiciones profesionales en Georgia con la formación de nuevas empresas con apoyo del Fondo Semilla.

En clave de contexto 5, fortalecimiento de las cadenas de valor, junto con los Sistemas Universitario y de Investigación Tecnológica. 
Alianza Productiva local de veintisiete empresas miembro, entre otros, y como miembros plenos del consorcio; National Semiconductor, Ciena, Echostar, Broadcom, Nortel Networks, IDT Microcoating Technology BarcoView Cypress Movaz Networks BellSouth VeriSign Intracom Siemens-Dematic.

Roles de industrias asociadas: distinguimos los roles de: exploración; investigación directa para retroalimentación durante reuniones del Consejo de Asistencia Industrial; de articulación: aprovechar oportunidades de investigación específicas, con proyectos de comercialización y de difusión: seminarios y "días" Yamacraw.

Recursos: el capital semilla de Yamacraw de "cinco millones de dólares" fue creado por el Estado de Georgia dirigido a proveer capitales de riesgo para infraestructura, particularmente: comunicaciones de banda ancha, tecnología de semiconductores y procesamiento de contenidos.

\section{Discusión:}

Vemos más de un área que espera ser explorada e investigada para alcanzar un grado de generalización mayor de los resultados exhibidos en este artículo.

\section{1. Áreas de investigación futura}

La exploración de estructuras de red en cada país y en el subcontinente americano es un área fértil que promete resultados valiosos, en cualquiera de los sectores que abarca este estudio: tecnología, cadenas productivas y educación con sentido operativo.

Relación con la cultura de los países sudamericanos. Como mínimo es necesario revisar y cuantificar las distancias entre el modelo aquí bosquejado y las realidades de cada país. Este concepto de distancia acepta varios espacios de operacionalización; por sectores, cadenas de valor, prácticas implícitas o explícitas de los colectivos vinculados a cada espacio: científicos, tecnólogos, institutos y centros tecnológicos, empresarios, cámaras y asociaciones civiles, redes, etc.-

\section{Relación con el sistema político.}

Aunque no ocupa el foco de este trabajo, la esfera de lo político, con sus contradicciones, quiebres y conflictos, condiciona en grado elevado las necesarias alianzas que facilitan la actividad colaborativa, con la sinergia y la productividad que ellas conllevan. El análisis de este campo de poder en su interacción con las áreas tecnológica y productiva, arrojará mayor luz sobre las condiciones que 
deberán cumplirse para una fructífera relación entre los mismos La condición necesaria: visibilizar la importancia del espacio donde la innovación ocurre- los espacios focalizados en este escrito- , para introducir la temática en la agenda política de cada país.

Queda claro que los análisis e inferencias que se realizan de los casos estudiados actúan como un laboratorio de observaciones siguiendo marcos de referencia, como la construcción de la Unión Europea, y casos complejos y completos de interdependencia institucional al interior de cada nación y con el espacio internacional.

Lo que podemos apreciar es que, en condiciones de contextos similares entre países- principio de isomorfismo -, las acciones colaborativas descriptas en este texto contienen los ingredientes de las actividades de cooperación internacional propios de los formatos conocidos de integración regional.

3. Desafío de enriquecer la teoría. Lo dicho en los puntos anteriores y un repaso a la bibliografía existente plantea la necesidad de intensificar la formalización de nuevos conceptos - constructos- y aportes teóricos que ayuden a revelar el carácter idiosincrático - específico e identitario de nuestra cultura- con relación al comportamiento colaborativo. A modo de ejemplo, la iniciativa Desafíos del Futuro del Senado de Chile - vista como un fractal reproducible en otros países- llevada adelante en estos últimos 4 años en sendos Congresos de 2012 a 2015, y orientada a la identificación sistemática de escenarios de futuro en el contexto latinoamericano, constituye un ejemplo de este reto. Imaginando posibles respuestas a este desafío, es factible pensar en la capacidad multiplicadora de internet a través de foros virtuales y presenciales que amplíen el alcance de este debate.

\section{Conclusiones}

Retomando la introducción postulamos que es imprescindible tanto registrar y mostrar casos germinales de conductas colaborativas en los diversos espacios de construcción institucional y, como ha quedado claro, hilándolas en sintonía con las herramientas del pensamiento sistémico. A modo de corolarios que se infieren de la conceptualización desarrollada aquí y de las interpretaciones que desplegamos en el relato de los casos, concluimos que:

1 - Está claro que el análisis de la complejidad -y los procesos de integración regional lo son en grado elevado- necesita del aporte de científicos, tecnólogos, investigadores y ciudadanos comprometidos de todas las áreas para aplicar esfuerzos colaborativos en la reunión de información dispersa o desconocida, gestionarla como nuevo conocimiento, con sentido de motivar acciones colectivas que 
muestren avances en el acercamiento de nuestras comunidades.

2 - En los casos estudiados se observan prácticas con abundancia de fractales organizacionales y colaborativos: redes formales e informales entre actores personales e institucionales, visión compartida de los colectivos involucrados, liderazgo por reconocimiento social y líderes por méritos tecnológicos y profesionales, planes estratégicos a 5 y más años. Ellos son ejemplos muy completos de alineamientos entre actores, de elevada capacidad para integrarse en iniciativas macro-regionales. Al mismo tiempo portan dispositivos asociativos relevantes y con potencial de suceso, como hemos intentado mostrar.

\section{3 - Sobre adaptación a contextos colaborativos}

Cada sistema vive del intercambio con los contextos analizados; el micro incluye el concepto existencial en que el hombre, al organizarse, genera microestrategias - implícita o explícitamente -, que despliega como ciudadano en varios espacios de acción meso, sea en el estado, en la actividad privada o en las organizaciones de la sociedad civil. El meso integra, a su vez y de modo simultáneo, el movimiento proyectual de cada ciudadano, organiza y potencia sus actores institucionales, los proyectos que promueven y lo eleva hacia el sistema macro. Esto implica recorrer el circuito comunicación/procesar información/ interesar a involucrados/ y orientar la acción efectiva en los cinco contextos relacionantes. También prever el impacto posible: así, ante una reunión de expertos sobre un proyecto de innovación tecnológica - fractal/área micro- , cómo podrá canalizarse en los planos meso - instituciones-, y en el nivel macro, en cuanto a contactar aliados para su desarrollo. Debe entenderse este ciclo en su dinámica sistémica que incluye: acción sistemática de presión acumulativa, pautada en tiempos, con logro de compromisos parciales y ampliando los contextos de escucha para el logro del resultado final. El caso Yamacraw sintetiza está dinámica de movilización de actores diversos.

4- Los drivers son imprescindibles para la actividad colaborativa, especialmente en las fases de mayor complejidad, como son las alianzas y asociaciones en el espacio internacional. En este marco, un vínculo estable de interacciones intensas en el espacio local-nacional-internacional, actúa como un campo de áreas continuas, que se interrelacionan de modo sistemático y permanente, alentando la sinergia entre las partes y la recursividad- o reproducción en otros espacios geográficos- como resultado natural. El caso de la acción de INATEL, actuando sobre las autoridades federales -Minas Gerais- y las nacionales, junto a una estrategia de aprendizaje de experiencias internacionales- Vía 
cooperación GTZ-Alemania durante 10 años, habla de este proceso.

5 - Los campos cultural y político permean los contextos y condicionan el grado de alcance y sustentabilidad que puede obtener cada emprendimiento colaborativo. Uno y otro favorecen la evolución de iniciativas cuando una cultura abierta y proactiva se conjuga con alineamientos políticos promotores de desarrollo a escala regional. 


\section{Referencias bibliográficas}

AUTOR. (2014). Tesis Doctoral. Gestión colaborativa del Conocimiento como Aceleradora de la Integración Regional. Universidad del Salvador.

BEST, E. (1993). Sistemas Institucionales para la integración Regional, en Los desafíos de la Integración: capacidades de respuesta a nivel gerencial, legal e institucional. CEFIR. Uruguay.

BOBBIO, N; (2006). Estado, gobierno y sociedad. Méjico: Ed. Fondo de Cultura Económica, pp. 81 a 85.

BRONFENBRENNER, U. (1987). La ecología del desarrollo humano. Barcelona: Ed. Paidós. BOURDIEU, P. 2003. Campo de poder, campo intelectual. Buenos Aires: Ed. Quadrata. BURT, R. (1995). The Structural Holes. Massachusetts: Ed. Harvard University Press. CARLSEN, S. (1997). Conceptual Modeling and Composition of Flexible Workflows Models PhDthesis. Trondheim, Noruega: NTNU- Norwegian University of Science and Technology. CASTELLS, M. (2001). La galaxia Internet. Reflexiones sobre Internet, empresa y sociedad", Barcelona: Plaza y Janés Editores.

HENRIC-COLL, Michel (2014). “La organización fractal”, en http://www.fractalteams.com/ebook fractalteams/organizacion-empresarial-sigloXXI-fractalteams.html; tomado de internet 16.10.2014 INATEL. (2009). Plan estratégico del POLO Tecnológico. Minas Gerais: Ed. INATEL. INATEL. (2002). Sonho e Realidade. Minas Gerais: Inatel, p.25.

INTI. (2008). Plan Estratégico. Buenos Aires: Ed. INTI.

NONAKA, I. \& TAKEUCHI (1999). La organización creadora de conocimiento. Méjico: Oxford University Press.

OYARZÚN SERRANO Lorena (2008). Sobre la naturaleza de la integración regional: teorías y debates* Revista de Ciencia Política / Volumen 28 / N² 95 -113, Univ. Católica de Chile. REAL ACADEMIA ESPAÑOLA. En URL: http://lema.rae.es/drae/?val=dispositivo; consultado el 08.08.2014.

ROMERO PÉREZ, CLARA, (2006). Paradigma de la complejidad, modelos científicos y conocimiento educativo. Visto en: ttp://www.uhu.es/agora/version01/digital/numeros/06/06articulos/monografico/html_6/clara_romero.htm ROMERO PÉREZ, C. (2001). El constructivismo cibernético como metateoría educativa: aportaciones al estudio y regulación de los procesos de enseñanza y aprendizaje, Teoría de la 
Educación. Sociedad y Cultura en la Sociedad de la Información, 3 (Revista Electrónica) http:// www3.usal.es/teoriaeducacion.

SCHWANDT, T. (1994). Contructivist, interpretivist persuassions for human inquiry, Handbook of qualitative research (pp. 118-137) Thousand Oaks, CA, Sage Publ.

SECTES, (2009) Secretaria de Estado de Ciencia, Tecnología e Ensino superior -- "Gestao Estratégica - Plan Director 2009-2013”. pp.19-25.

SECTES, (2010). Minas Avanca na economía do Conhecimento. Minas Gerais: Ed. FAMEPIG.

SECTES (2010). -Secretaria de Estado de Ciencia, Tecnología e Ensino Superior - Plan Estratégico de Gobierno Minas Gerais 2010-2014.

SENADO DE CHILE, Congreso del Futuro, 2012 a 1015. http://www.senado.cl/inscripción-ivcongreso-del-futuro/prontus_senado/2014-11-04/175752.html

SENGE, P. (1998). La Quinta disciplina. Ed. Granica, Barcelona. España.

STAKE, R. (1999). Investigación con estudio de casos. Madrid: Ediciones Morata S.L

VON BERTALANFFY, (1995). Teoría General de los Sistemas. Méjico, Fondo de Cultura Económica.

VON FOERSTER, H. (1996). Las semillas de la cibernética. Obras escogidas. Barcelona: Gedisa. VON HALDENWANG, Christian (2005). Integración regional en América Latina. La perspectiva de la gobernabilidad. Revista Nueva Sociedad, № 195. 